Port Acadie

Revue interdisciplinaire en études acadiennes

An Interdisciplinary Review in Acadian Studies

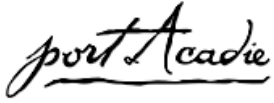

\title{
Les sources ethnomusicologiques des Arts et Traditions Populaires aux Archives nationales, valorisation numérique d'un patrimoine unique
}

\section{Martine Sin Blima-Barru et Pascal Riviale}

Numéro 30, automne 2016

Patrimoine oral et valorisation à l'ère du numérique ( $1^{\text {ère }}$ partie)

URI : https://id.erudit.org/iderudit/1056921ar

DOI : https://doi.org/10.7202/1056921ar

Aller au sommaire du numéro

Éditeur(s)

Université Sainte-Anne

ISSN

1498-7651 (imprimé)

1916-7334 (numérique)

Découvrir la revue

Citer cet article

Blima-Barru, M. S. \& Riviale, P. (2016). Les sources ethnomusicologiques des Arts et Traditions Populaires aux Archives nationales, valorisation numérique d'un patrimoine unique. Port Acadie, (30), 77-89.

https://doi.org/10.7202/1056921ar
Résumé de l'article

Pendant cinq décennies Claudie Marcel-Dubois et Maguy Andral, figures incontournables de l'ethnomusicologie au Musée des Arts et Traditions populaires, collectent un matériel d'étude d'une grande richesse et d'une grande diversité, abordant les pratiques musicales, la facture et l'organologie sur la presque totalité du territoire métropolitain français et dans certaines terres d'outre-mer. À la veille de redéployer ses collections à Marseille, le Musée des Arts et Traditions populaires (devenu MuCEM en 2005) lance une vaste opération de sauvegarde des archives. L'article apporte un éclairage particulier sur les archives ethnomusicologiques qui tiennent, dans cet ensemble, une place éminente. Dans le cadre d'un partenariat entre les Archives nationales, une équipe du IIAC (laboratoire d'anthropologie CNRS-EHESS) et MuCEM, la numérisation des archives écrites, des photographies et des enregistrements sonores, est en voie d'achèvement, prochainement accessibles sur Internet. L'éventail des possibilités d'interrogation et d'exploitation qu'ouvrira la dématérialisation d'un fonds sans équivalent pour l'ethnomusicologie en France est très vaste. 


\title{
Les sources ethnomusicologiques des Arts et Traditions Populaires aux Archives nationales, valorisation numérique d'un patrimoine unique
}

\author{
Martine Sin Blima-Barru \\ Archives nationales (France) \\ Pascal Riviale \\ Archives nationales (France)
}

\begin{abstract}
Résumé
Pendant cinq décennies Claudie Marcel-Dubois et Maguy Andral, figures incontournables de l'ethnomusicologie au Musée des Arts et Traditions populaires, collectent un matériel d'étude d'une grande richesse et d'une grande diversité, abordant les pratiques musicales, la facture et l'organologie sur la presque totalité du territoire métropolitain français et dans certaines terres d'outre-mer. À la veille de redéployer ses collections à Marseille, le Musée des Arts et Traditions populaires (devenu MuCEM en 2005) lance une vaste opération de sauvegarde des archives. L'article apporte un éclairage particulier sur les archives ethnomusicologiques qui tiennent, dans cet ensemble, une place éminente. Dans le cadre d'un partenariat entre les Archives nationales, une équipe du IIAC (laboratoire d'anthropologie CNRS-EHESS) et MuCEM, la numérisation des archives écrites, des photographies et des enregistrements sonores, est en voie d'achèvement, prochainement accessibles sur Internet. L'éventail des possibilités d'interrogation et d'exploitation qu'ouvrira la dématérialisation d'un fonds sans équivalent pour l'ethnomusicologie en France est très vaste.
\end{abstract}

\begin{abstract}
For five decades, Claudie Marcel-Dubois and Maguy Andral, key figures in ethnomusicology at the Museum of Popular Arts and Traditions, collected study material of great diversity, addressing musical practices and organology on almost all French territory, including some overseas territories. On the eve of reopening its collections in Marseille, the Museum of Popular Arts and Traditions (renamed MUCEM in 2005) launched a vast operation to safeguard the archives. The article sheds a particular light on the ethnomusicological archives which figure prominently in these collections. Thanks to a partnership between the National Archives, a team from the IIAC (CNRS-EHESS Anthropology Laboratory) and MUCEM, the digitization of the written archives, photographs and sound recordings is nearing completion and will soon be accessible on the Internet. The dematerialization of an unrivaled collection for ethnomusicology in France opens a broad range of new possibilities for examination and interpretation.
\end{abstract}




\section{Mots clés}

ethnomusicologie, archives sonores, Archives nationales (France), numérisation

\section{Keywords}

ethnomusicology, sound archives, National Archives (France), digitization

Entre 2012 et 2014 a été versée aux Archives nationales une partie importante des archives du Musée national des Arts et Traditions populaires (MNATP), aboutissement d'un vaste chantier mené par la mission des archives du Service interministériel des Archives de France auprès du ministère de la Culture de la Communication. Parmi celles-ci se trouvent les documents relatifs à la phonothèque et aux enquêtes ethnomusicologiques réalisées par le musée pendant près de cinquante ans. Ceux-ci ont fait l'objet - dans les derniers temps du MNATP ou bien plus récemment - de campagnes de numérisation, qui offrent pour la première fois des possibilités d'accès aux fonds à tous les publics, ouvrant ainsi de nouvelles perspectives de recherches et de valorisation.

Un retour en arrière s'impose : lorsqu'en 1928 le docteur Paul Rivet fut nommé directeur du Musée d'Ethnographie du Trocadéro, il entendait procéder à une réorientation de cette institution afin de mieux prendre en compte les nouvelles tendances de l'anthropologie culturelle. Dans cette perspective il s'adjoignit les services d'un personnage atypique dans ce milieu scientifique : Georges Henri Rivière ${ }^{1}$. Ensemble, ils entreprirent de concevoir une institution novatrice, combinant musée, bibliothèque, centre de recherche et lieu d'enseignement, qui aboutit finalement à l'éclatement du musée en deux parties distinctes en 1937. Tandis que le M.E.T. devenait le Musée de l'Homme, les collections ethnographiques et folkloriques françaises (qui formaient au musée depuis 1884 une "salle française ») servaient de noyau

1 Brice Gérard, Histoire de l'ethnomusicologie en France (1929-1961), Paris, L'Harmattan, coll. « Histoire des sciences humaines », 2015, 372 p. 
initial au Musée national des Arts et Traditions populaires ${ }^{2}$. Faute de moyens, ce musée fonctionna pendant plusieurs décennies dans des espaces contraints, dans les sous-sols de l'aile ouest du Palais de Chaillot (sous le Musée des Monuments français), dans l'attente d'un bâtiment construit spécifiquement pour lui. Ce bâtiment édifié à l'orée du bois de Boulogne, œuvre des architectes Jean Dubuisson et Michel Jausserand, ne fut achevé qu'en 1969. Une "galerie d'étude » fut d'abord ouverte en 1972, puis une " galerie culturelle» en 1975. Cependant, souffrant d'un certain éloignement de Paris, d'un manque de renouvellement et d'un désintérêt croissant du public pour les thématiques folkloriques et ethnologiques, le musée traversa une longue crise qui aboutit à sa fermeture en 2005 et au transfert de ses collections au MuCEM, inauguré à Marseille en juin $2013^{3}$.

En 2010 le MuCEM fit appel à la mission des archives du ministère de la Culture et dela Communication afin de l'aider à traiter la question de l'archivage des données de l'ancienne institution. Le récolement des fonds effectué par elle signalait alors un ensemble de plus de 1300 mètres linéaires de documents souvent dispersés, mal identifiés, parfois peu aisément accessibles, où se mêlaient archives et documentation. Le long travail de la mission consista alors à reconstituer des ensembles cohérents, tant d'un point de vue administratif qu'intellectuel, d'en faire la description archivistique, puis de procéder au reconditionnement des documents en vue de leur transfert aux Archives nationales. Les premiers versements commencèrent en 2012, avant de s'échelonner à un rythme soutenu tout au long de l'année 2013, pour s'achever au premier semestre 2014. Ce sont au total 66 versements qui auront été effectués

2 Un décret du $1^{\mathrm{er}}$ mai 1937 institua un département des arts et traditions populaires au sein de la section des beaux-arts du ministère de l'Instruction publique ; ce décret prévoyait « un musée installé au Palais du Trocadéro et des musées de plein air qui seront organisés dans des domaines de l'État sur divers points du territoire ». Seul le premier vit le jour.

3 Pour un historique du MNATP, lire Nina Gorgus, Le magicien des vitrines : le muséologue Georges-Henri Rivière, Paris, Éditions de la Maison des Sciences de l'Homme, 2003, 416 p., et Martine Segalen, Vie d'un musée, 1937-2005, Paris, Stock, coll. «Un ordre d'idées », 2005, 360 p. 
(57 sur le site de Pierrefitte-sur-Seine, neuf sur celui de Fontainebleau), représentant environ 4000 articles sur tous types de supports et de tous formats : papiers (liasses, fiches ou registres), cartes, plans et autres documents figurés (sur papier ou calque, manuscrits ou imprimés), affiches, photographies, films, enregistrements sonores, objets divers. À la demande du MuCEM, qui souhaitait numériser plusieurs ensembles de documents, une partie du fonds d'archives a en outre été prise en dépôt par la nouvelle institution marseillaise ${ }^{4}$. D'autres documents ont également rejoint le MuCEM constituant une partie de leur collection muséale ou de leur documentation comme une part importante des photographies et les films donnés ou reçus par le MNATP5.

Ce vaste ensemble d'archives constitue un fonds exceptionnel, tant par sa richesse que par sa cohérence. Il nous permet bien sûr en premier lieu de documenter précisément les activités muséographiques de l'ancien musée national des Arts et Traditions populaires. On dispose ainsi des registres d'entrées des collections d'objets $^{6}$, mais aussi des registres où sont répertoriés les manuscrits, les documents figurés, les films, les enregistrements sonores, etc. Parmi les autres documents directement liés à l'activité muséographique du MNATP, citons les dossiers d'expositions, les affiches, ainsi que les prêts aux expositions. Enfin, on dispose de

4 Une convention a été signée par le Service interministériel des archives de France, les Archives nationales et le MuCEM, dans laquelle cet établissement s'engage à verser progressivement (au fil de l'avancement des opérations de numérisation) ces documents aux Archives nationales pour qu'ils puissent également être mis à la disposition des chercheurs. Ce dépôt comprend les archives écrites d'une grande partie des enquêtes et enquêtes-collectes menées par le MNATP. Les enquêtes musicales ne sont pas concernées par ce dépôt.

5 À noter que la plupart des négatifs sont conservés au MuCEM (la photothèque du MNATP ayant suivi les collections à Marseille) ; la base PhoCEM permet de visualiser un grand nombre d'images produites au cours des enquêtes ou bien en relation avec les collections ou la scénographie du musée (http://www.culture. gouv.fr/public/mistral/phocem_fr).

6 Établis tout d'abord rétrospectivement pour les premiers objets entrés au musée d'ethnographie du Trocadéro depuis 1881, ces registres - qui vont jusqu'au milieu des années 1950 -, ont fait l'objet d'une campagne de numérisation en 2013. 
toute la documentation relative à l'organisation interne du musée : le personnel, la bibliothèque, les archives, le bâtiment.

Le MNATP était novateur à plus d'un titre ; l'une des fiertés de son créateur Georges Henri Rivière était d'avoir inspiré le concept d'un établissement muséal qui se trouverait au cœur de la recherche: initiant toujours de nouvelles études sur le terrain, présentant sur le plan muséographique les premiers résultats, tout en reformulant éventuellement ses questionnements en fonction de nouvelles problématiques de recherches. Dès sa création le musée fut placé au centre d'un dispositif d'enquêtes de terrain destiné à réunir un large corpus d'informations sur le "folklore » français, enquêtes au cours desquelles des matériaux d'étude sur les sujets les plus divers furent collectés (chants et contes traditionnels, données sur l'habitat, le mobilier, les vêtements, les coutumes locales, etc.).

Les archives versées permettent de comprendre comment étaient organisées ces missions d'enquête, quels en étaient les résultats, comment ceux-ci étaient - ou pas - utilisés. On voit aussi comment au fil du temps le MNATP a suivi une évolution tout à fait notable dans ce cadre-là, passant des premières enquêtes folkloriques, aux chantiers intellectuels sous l'Occupation, puis aux enquêtes systématiques de l'après-guerre pour documenter les cultures locales et régionales, ainsi qu'aux enquêtes-collectes ; de même le musée formalisa une longue association au CNRS avec la création du Centre d'Ethnologie Française. Entre 1964 et 1968, dans le cadre des Recherches Coopératives sur Programme (RCP), deux enquêtes sont également organisées par le CNRS et avec le concours du MNATP ${ }^{7}$, reposant sur l'étude pluridisciplinaire de deux régions : l'Aubrac et le Châtillonnais, ainsi que la collecte d'objets. La reconstitution du « buron de l'Aubrac » (ferme où se fabrique le fromage), née de la collecte, devient un point fort de la muséographie de Georges Henri Rivière, dans la Galerie culturelle où il est exposé. Comme le déclarait Georges Henri Rivière lors d'un congrès en 1964 :

7 Dans les années 1960, le CNRS lance plusieurs campagnes de collectes et de conservations des « vestiges » du passé. Le MNAP est associé à deux d'entre elles. 
La recherche est l'infrastructure de toute notre institution. Elle constitue notre contribution à l'avancement de l'ethnologie, elle conditionne le rassemblement des objets et des documents, elle inspire l'action éducative et culturelle. Nous sommes en définitive un musée-laboratoire et cela explique que le Centre national de la recherche scientifique nous donne son appui ${ }^{8}$.

Les archives relatives à ces diverses enquêtes fournissent par conséquent une formidable documentation pour servir à l'historiographie de l'ethnographie française'. Entre 1970 et 2005, les enquêtes-collectes se poursuivent sur des thématiques plus larges. Parmi celles-ci se distinguent celles sur le cirque et la fête foraine (Zeev Gourarier, Jacqueline Christophe), sur le SIDA (Françoise Loux), sur le hip-hop et le graffiti (Claire Calogirou), sur les musiques amplifiées (Marc Touché). Elles contribuent largement à l'enrichissement des collections du musée.

Le musée a été particulièrement actif dans le domaine de l'ethnomusicologie, par l'entremise de deux chercheuses du CNRS, intimement associées à l'institution muséale en tant que chargées de missions et de responsables de la phonothèque, Claudie-Marcel Dubois (1913-1989) et Maguy Pichonnet-Andral (1922-2004). Soit en tandem - le cas le plus fréquent -, soit seules, elles ont mis en

8 Intervention de G.H. Rivière lors d'un congrès à Moscou en août 1964 (Ségolène, op. cit., p. 173). En outre une bonne partie du personnel du musée était constituée de personnes détachées du CNRS.

9 Sur ces enquêtes la bibliographie est abondante. Voir notamment, Bertrand Müller et Florence Weber, « Réseaux de correspondants et missions folkloriques. Le travail d'enquête, en France, vers 1930 », dans Gradhiva, n 33, 1993, p. 4355 ; Marie-Noëlle Denis, « L'enquête d'architecture rurale (1940-1968), une étape dans la construction de l'ethnologie française », dans Du folklore à l'ethnologie sous la direction de Jacqueline Christophe, Denis-Michel Boëll et Régis Meyran, Paris, Maison des sciences de l'Homme, 2009, p. 49-61 ; Pascal Riviale, « Des études en quête d'auteurs : instructions et questionnaires sur l'habitat traditionnel en France, fin XIX ${ }^{\mathrm{e}}$-milieu XX ${ }^{\mathrm{e}}$ siècle », dans In Situ, $\mathrm{n}^{\circ} 30$ (" Au regard des métiers du patrimoine »), 2016 ; François Gasnault et Marie-Barbara Le Gonidec, "Enquêter en tandem sur les pratiques musicales de la France rurale pour le Musée des arts et traditions populaires : variation ou conjuration du collectif ?", dans Ethnographiques.org, ${ }^{\circ}$ 32, "Enquêtes collectives », septembre 2016. En ligne : http://www.ethnographiques.org/2016/Gasnault,Le-Gonidec. 
place et effectué pendant plus d'une quarantaine d'années - de 1939 à 1986 - des campagnes d'enquêtes et de collectes sonores liées aux traditions musicales francophones, d'abord sur le territoire métropolitain, puis à partir de 1955 dans quelques pays étrangers ou dans plusieurs régions outre-mer ${ }^{10}$.

Les archives du MNATP versées aux Archives nationales en 2013 constituent évidemment des sources essentielles pour documenter ce travail et en suivre les évolutions au fil du temps et de l'expérience des enquêtrices et des enquêteurs : évolution dans l'approche elle-même de l'objet d'étude (passant du « folklore musical » au " phénomène musique », Marie-Barbara Le Gonidec et François Gasnault 2016 : 3), mise en place d'une méthodologie bien rodée, etc.

Les archives des enquêtes ethnomusicologiques du MNATP sont réunies dans un ensemble portant aux Archives nationales les numéros de versement 20130043 (archives écrites), 20130007 et 20130009 (archives sonores des enregistrements de terrain), 20130042 et 20130068 (certains articles de ces versements sont les films réalisés au moment des enquêtes de terrain). La première partie du versement 20130043 est plus spécifiquement dédiée à la gestion et à l'organisation des enregistrements sonores qui étaient conservés à la phonothèque du musée : on y trouve les registres d'inventaire des collections sonores, ainsi que des dossiers documentaires (avec documentation diverse et correspondance) relatifs aux enregistrements sonores entrés au musée autrement que par les campagnes programmées par l'établissement. Les archives administratives du musée sont conservées dans le versement 20130520. La partie audiovisuelle, issue de la collecte des enregistrements sonores et des films fixant la transmission des chants, airs et récitations des "informateurs », était également conservée à la phonothèque. Outre les captations réalisées au cours

10 La première incartade au territoire métropolitain se situe en Sicile en 1955, puis seront effectuées des missions en Gaspésie/Québec (1955 et 1961), Tchécoslovaquie (1957), Mexique (1962), îles du Canada oriental (1962-1963), îles anglo-normandes (1970), Canada (1970), Suisse romande (1972), Antilles (1978 et 1982), Réunion (1979). 
des missions sur le terrain, la phonothèque du MNATP enrichissait ses collections de diverses façons : par le biais de dons et d'envois faits par des correspondants ou des confrères ethnologues travaillant sur d'autres terrains, ou bien par des enregistrements faits à Paris à l'occasion du passage de musiciens en relation avec le musée ${ }^{11}$. Le deuxième grand ensemble est constitué par les dossiers relatifs aux missions d'enquêtes menées sur le terrain à l'instigation des musicologues du MNATP.

La typologie des documents conservés pour chacune de ces enquêtes est presque toujours identique, ce qui permet de comprendre comment elles étaient organisées et ce que l'on peut espérer y trouver comme type d'informations. Globalement, les dossiers des missions s'articulent en quelques grands sousdossiers : l'organisation, le travail sur le terrain, la documentation secondaire collectée, la clôture de la mission. Dans la partie dédiée à l'organisation de l'enquête on trouvera donc essentiellement des correspondances (avec des correspondants locaux ${ }^{12}$, qu'il s'agisse de particuliers, d'institutions - musées, universités, etc. -,

11 Dans un de ces dossiers on peut ainsi lire un courrier envoyé à Claudie Marcel-Dubois par Gaston Rivière, " accordéoniste, vielleux, cornemuseux, diseur paysan ", l'informant d'un prochain passage à Paris : "Suite à nos correspondances au sujet d'enregistrements, j'ai un engagement pour Paris le samedi 18 février. Il me serait possible de partir le jeudi et de vous disposer le vendredi 17 le temps nécessaire à ce travail. » (20130043/16 : dossier « 56.1 : Bourbonnais, vielle à roue et cornemuse », lettre du 21 décembre 1955).

12 Et l'on voit là l'importance accordée dès les premiers temps du musée aux réseaux de relations tissés par l'institution ou -à titre plus personnel - par ses chercheurs. Les archives du MNATP en sont aussi le reflet. Nous renvoyons le lecteur notamment aux versements suivants : 20130043 : dossiers relatifs aux archives sonores inédites (1939-2013) ; 20130098 : relations avec des institutions publiques, associations et organismes à vocation culturelle et pédagogique (1894-2009) ; 20130147 : archives des ressources documentaires et activités scientifiques du Musée des Arts et traditions populaires (1908-2011) ; 20130218 (activités de Jean Cuisenier, directeur du Centre d'ethnologie française, 1963-1987) ; 20130277, 20130521 et 20130648 (enquêtes réalisées dans le cadre des chantiers intellectuels français et autres enquêtes menées par le musée ou par des institutions antérieures, 1899-2006) ; 20130452 (Fonds Marcel-Maget : dossier personnel, enquêtes et enseignement, 1902-1993) ; 20130478 (documents produits dans le cadre des séminaires, colloques et des relations du MNATP avec les institutions françaises et étrangères, 1970-2007). 
d'associations culturelles), où l'on voit se dessiner et se préciser le projet d'enquête, ses objectifs, les personnes à contacter sur place. On y trouvera également des notes scientifiques et administratives destinées à la hiérarchie et à la tutelle en vue d'obtenir les crédits et le matériel nécessaires. La partie la plus conséquente du dossier pour chaque mission est celle portant sur le travail sur le terrain, avec des journaux de route (surtout pour les premières années), des carnets relatifs aux informateurs, des carnets de notes sur les chants enregistrés, des transcriptions (paroles et musique), les minutes d'enregistrement des clichés réalisés au cours de la mission, ainsi qu'un grand nombre de tirages papiers de ces photographies. À l'occasion de leurs travaux sur le terrain, il n'était pas rare que les enquêtrices collectent également des informations sur des costumes traditionnels, des cérémonies, des instruments de musique ou leurs fabricants : ainsi dans le dossier relatif à une mission dans la vallée de la Sioule (Allier) en 1963, trouve-t-on une très intéressante série de croquis faits dans l'atelier du luthier Pajot à Jenzat ${ }^{13}$. De la même manière, au cours de ces missions étaient aussi collectés à titre documentaire des publications diverses (histoires locales, guides touristiques, brochures d'associations, programmes de festivités), des cartes postales ainsi que des tirages anciens offerts par des informateurs (notamment des noces ou des groupes folkloriques photographiés entre les années 1920 et les années 1960). Enfin, un dernier dossier évoque la clôture de la mission, avec des rapports, des correspondances et des projets de publications (réalisées ou non ${ }^{14}$ ).

Les enregistrements sonores de toutes les enquêtes de terrain ont subi les contraintes matérielles des technologies utilisées pour fixer les captations, forcément soumises à la forte évolution technique de ce secteur, d'autant plus forte que les enquêtes s'étalent sur le temps long. Des pyrals initialement utilisés, les

13 Ces dessins sont conservés aux Archives nationales dans l'article 20130043/84.

14 Selon Le Gonidec et Gasnault (op. cit.) ce matériel ethnographique collecté durant plusieurs décennies aurait finalement donné lieu à un nombre assez réduit de publications, ce qui rend ces archives d'autant plus intéressantes tant pour les historiens, que pour les ethnologues et les musiciens d'aujourd'hui. 
chercheurs sont passés aux cassettes audio. Le support impacte forcément l'acte d'enregistrer, le plaçant dans un espace temporel standard qui impose de fréquentes coupures dans les interventions des musiciens et chanteurs. Au moment où ces documents ont été numérisés par le MNATP, l'archive initiale a été respectée. Les fichiers numérisés reproduisent donc l'alternance des faces A et faces B des enregistrements analogiques, en préservant l'intégrité des archives. Au moment du transfert des archives audiovisuelles aux Archives nationales, les fonds ont été regroupés par typologie de supports. Les pyrals ont été réunis ; les enregistrements issus des collectes de terrain sur cassettes audio ont été regroupés avec d'autres enregistrements utilisant ce même type de support (enquêtes réalisées par d'autres chercheurs que ceux du MNATP, au cours du séminaire de l'EHESS-EPHE). Les films et vidéos ont été divisés entre productions effectuées par des acteurs hors MNATP (transférés au MuCEM) et les réalisations du musée (transférées aux Archives nationales), mêlées aux films réalisés au moment d'exposition ou des enregistrements institutionnels liés à des événements. Le lien entre les archives écrites ou audiovisuelles reste, pour les collectes de terrain, le numéro inscrit dans les registres d'inventaire de la phonothèque du musée qui identifie une même enquête par l'année de sa réalisation, ou de son enregistrement à la phonothèque, et un numéro d'ordre.

Durant la première partie de la vie du MNATP, lorsqu'il était installé dans les sous-sols du Palais de Chaillot (sous le musée des Monuments français), le musée disposait de très peu d'espaces et n'était donc pas en mesure de faire des présentations permanentes de ses collections qui ne cessaient de s'accroître depuis sa fondation. Ce n'est qu'en 1951 que les travaux d'aménagement des salles d'expositions temporaires furent achevés, autorisant enfin le lancement d'une longue série d'expositions permettant l'exploitation scientifique et la valorisation de ces collections demeurant en réserve. Ces expositions utilisent parfois les données et les objets collectés lors des enquêtes musicales. Tel fut par exemple le cas avec la toute première exposition organisée dans les 
locaux du MNATP du Palais de Chaillot (du 23 juin au 22 octobre 1951) : «Bretagne, problèmes d'ethnographie régionale et objets d'art populaire », exposition collective qui comptait Claudie MarcelDubois et Marguerite Pichonnet-Andral parmi ses commissaires. Quelques vues photographiques des vitrines et de la scénographie montrent l'importance qui était accordée aux instruments de musique traditionnels dans cette exposition ${ }^{15}$; d'ailleurs, selon Martine Segalen, des enregistrements de musique bretonne étaient diffusés dans les salles d'exposition ${ }^{16}$. Bien plus tard, dans le nouvel édifice du MNATP du bois de Boulogne, deux expositions furent consacrées spécifiquement aux instruments et aux pratiques musicales traditionnelles : « L'instrument de musique populaire. Usages et symboles » (novembre 1980-avril 1981) et "Souffler c'est jouer » (avril à août 1999). Les archives sonores étaient donc très peu utilisées, restant encore largement inconnues. De plus, seul un petit nombre d'enregistrements ont fait l'objet d'une édition : le MNATP n'a publié que quatorze extraits d'enquêtes entre 1949 et 2002. Le réseau des associations réparti dans les régions de France a par contre sollicité le MNATP pour disposer, dans le cadre de conventions de partenariat, de copies des enregistrements sonores qui les concernaient.

Parmi les associations qui ont demandé la mise à disposition d'enregistrements, on peut citer DASTUM, le Centre occitan des musiques et danses traditionnelles Toulouse Midi-Pyrénées, les Archives départementales de la Manche, la Maison méditerranéenne des sciences de l'homme de l'Université de Provence, le Centre des musiques traditionnelles de Rhône-Alpes, le Conseil général des Pyrénées atlantique, le Centre des musique et danses traditionnelles contemporaines et populaires de Guadeloupe, le Centre international de Musique populaire et l'Institut d'études occitanes du Limousin.

15 Le dossier de cette exposition est conservé sous les cotes 20120297/52 et 53. Les expositions suivantes se trouvent dans ce même versement ou bien dans ceux numérotés 20120397 et 20130219. 
Enfin, les archives du service de la phonothèque ${ }^{17}$ donnent quantité d'informations sur la gestion, l'étude, la valorisation et la diffusion des collections d'enregistrements sonores (notamment par le biais de dépôts dans des centres d'archives en région ou bien auprès d'associations), les inventaires et les campagnes de collectes, en particulier dans le cadre d'enquêtes-collectes menées à partir des années 1960. Puisque l'on traite ici de la question des sources ethnomusicologiques du MNATP, il convient de rappeler, par souci d'exhaustivité, que d'autres versements seraient utiles pour documenter plus complètement les activités musicales de cet établissement. En effet, les Archives nationales conservent également un jeu des registres d'inventaire ${ }^{18}$ des collections d'objets entrés entre 1881 et 1955, ainsi que les archives du service des collections.

C'est, on le voit, une masse considérable de sources diverses aujourd'hui conservées aux Archives nationales, qui sont disponibles, tant pour la recherche historiographique sur l'ethnomusicologie française que dans la perspective de retrouver un patrimoine musical parfois oublié. Depuis 2015, les Archives nationales, en partenariat avec le Laboratoire d'anthropologie et d'histoire de l'institution de la Culture (CNRS/EHESS, IIAC [Institut interdisciplinaire d'anthropologie du contemporain]-LAHIC [Laboratoire d'anthropologie et d'histoire de l'institution de la culture]), ont entrepris la mise à disposition d'archives du MNATP en utilisant la valorisation numérique, comme seule permettant de recréer la cohérence intellectuelle et de restituer la démarche des chercheurs. Ce partenariat s'appuie sur le programme développé par le LAHIC au sein de l'axe Culture du IIAC-LANIC sur les « Sources, Archives et Histoire Institutionnelle de l'Ethnomusicologie de la France (SAHIEF) » dont les archives de l'ancien MNATP sont le support. Dans ce cadre la numérisation des enquêtes de terrain est répartie

17 Les archives du service de la phonothèque sont conservées dans le versement 20130520.

18 Ces registres conservés sous les cotes 20130297/1 à 39 ont été intégralement numérisés. Les archives du service des collections correspondent au versement 20140106. 
entre archives écrites intégrées par les Archives nationales dans son marché pluriannuel de numérisation, et archives photographiques numérisées par le MuCEM. Les Archives nationales sont actuellement en train d'intégrer ces images à ses instruments de recherche pour rendre accessible la partie des enquêtes-collectes constituée par la documentation papier et les photographies dans la salle des inventaires virtuelle des Archives nationales sur son site Internet.

En 2016, le partenariat a connu une étape importante dans le cadre d'un accord de soutien à la production de contenus diffusés par le portail PSL-Explore et la Toile des savoirs PSL : Les Réveillées. L'usage de ce vocable semble en effet particulièrement approprié pour qualifier ces enquêtes ethnographiques restées longtemps peu accessibles. Poursuivant le programme de recherche du SAHIEF, Les Réveillées, porté par le LAHIC, doit permettre de rassembler, éditer et rendre accessibles dans une plate-forme d'éditorialisation, les corpus d'archives multimédias (textuelles, graphiques, sonores, photographiques et même, pour une très modeste part, audiovisuelles) correspondant aux quelques quarante enquêtes ethnomusicologiques. Cette valorisation permet pour la première fois de mettre en relation des archives de formes différentes, conservées parfois dans des institutions patrimoniales distinctes.

L'objectif est de favoriser l'édition scientifique des enquêtes ethnomusicologiques de terrain réalisées entre 1939 et 1986 afin de pouvoir diffuser largement les matériaux auprès de la communauté des chercheurs et d'en permettre sa restitution à un plus large public. 